Homology, Homotopy and Applications, vol.4(2), 2002, pp.17-27

\title{
HOMOTOPY LIE ALGEBRAS AND POINCARÉ SERIES OF ALGEBRAS WITH MONOMIAL RELATIONS
}

\author{
LUCHEZAR L. AVRAMOV \\ (communicated by Clas Löfwall)
}

\begin{abstract}
To every homogeneous ideal of a polynomial ring $S$ over a field $K$, Macaulay assigned an ideal generated by monomials in the indeterminates and with the same Hilbert function. Thus, from the point of view of Hilbert series residue rings modulo monomial ideals display the most general behavior. The homological perspective reveals a very different picture. Two aspects are particularly relevant to this paper:

If $I$ is generated by monomials, then the Poincare series of the residue field $k$ of $S / I$ is rational by Backelin [7], and the homotopy Lie algebra of $S / I$ is finitely generated by Backelin and Roos [8]. Constructions of Anick [1] and Roos [15], respectively, show that these properties may fail for general homogeneous ideals.

Recenly, Gasharov, Peeva, and Welker [12] showed that some homological properties of $S / I$, such as being Golod, depend only on combinatorial data gathered from a minimal set of monomial generators.

Here we prove that these data determine the Poincaré series of $k$ over $S / I$, along with most of its homotopy Lie algebra. As a consequence, we obtain the surprising result that if the number of generators of the ideal $I$ is fixed, then the number of such Poincaré series is finite, even when $K$ ranges over all fields.
\end{abstract}

\section{To Jan-Erik Roos on his sixty-fifth birthday}

\section{Results}

Let $K$ be a commutative ring and $\boldsymbol{x}$ a finite set of indeterminates over $K$. A monomial ideal in the polynomial ring $S=K[\boldsymbol{x}]$ is an ideal $I$ generated by some subset $M$ in $\boldsymbol{x}$. It is well known, and easy to see, that such an $I$ has a uniquely defined minimal (with respect to inclusion) set of monomial generators $M_{I}$.

Partly supported by a grant from the National Science Foundation.

Received February 15, 2001, revised May 8, 2002; published on July 12, 2002.

2000 Mathematics Subject Classification: 13D.

Key words and phrases: monomial ideal, graded Lie algebra, Poincaré series.

(C) 2002, Luchezar L. Avramov. Permission to copy for private use granted. 
For every $P \subseteq M_{I}$ set $m_{P}=\operatorname{lcm}\{p \mid p \in P\}$; thus, $m_{\varnothing}=1$, and $m_{\{p\}}=p$ for $p \in M_{I}$. The $L C M$ lattice, cf. [12], is the set $L_{I}=\left\{m_{P} \in S \mid P \subseteq M_{I}\right\}$ ordered by divisibility: $m_{P} \leqslant m_{Q}$ if and only if $m_{P} \mid m_{Q}$. The GCD graph $G_{I}$ has the elements of $L_{I}$ as vertices, and the pairs $\left\{m_{P}, m_{Q}\right\}$ with $\operatorname{gcd}\left\{m_{P}, m_{Q}\right\}=1$ as edges.

Set $R=S / I$ and $k=R /(\boldsymbol{x})$. The $K$-module $\operatorname{Ext}_{R}^{\circ}(k, k)$, graded by cohomological degree and equipped with Yoneda products, becomes a graded associative algebra. When $K$ is a field it is the universal enveloping algebra of a graded Lie algebra $\pi^{\bullet}(R)$, called the homotopy Lie algebra of $R$, cf. $[\mathbf{5}, \S 10]$ for details.

Theorem 1. Let $K$ be a field, let $\boldsymbol{x}$ and $\boldsymbol{x}^{\prime}$ be finite sets of indeterminates, let $I \subseteq K[\boldsymbol{x}]$ and $I^{\prime} \subseteq K\left[\boldsymbol{x}^{\prime}\right]$ be ideals generated by monomials of degree at least 2 , and let $R=K[\boldsymbol{x}] / I$ and $R=K\left[\boldsymbol{x}^{\prime}\right] / I^{\prime}$ be the corresponding residue rings.

If $\lambda: L_{I} \rightarrow L_{I^{\prime}}$ is an isomorphism of lattices inducing an isomorphism of graphs $G_{I} \rightarrow G_{I^{\prime}}$, then there is an isomorphism of graded Lie algebras over $K$,

$$
\pi^{\geqslant 2}(R) \cong \pi^{\geqslant 2}\left(R^{\prime}\right) .
$$

Let $n$ be a natural number. If $\boldsymbol{x}$ ranges over all finite sets of indeterminates and $I$ ranges over all ideals in $K[\boldsymbol{x}]$ generated by $n$ monomials in $\boldsymbol{x}$, then there exists only a finite number of isomorphism classes of graded Lie algebras $\pi^{\geqslant 2}(K[\boldsymbol{x}] / I)$.

A proof is presented Section 2. Here we record some corollaries.

Every finite $R$-module $N$ has a Poincaré series, defined to be the power series

$$
\mathrm{P}_{N}^{R}(t)=\sum_{j \in \mathbb{N}} \operatorname{rank}_{K} \operatorname{Ext}_{R}^{j}(N, k) t^{j} \in \mathbb{Z}[[t]] .
$$

The Lie algebra $\pi^{\bullet}(R)$ always determines the Poincaré series of $k$ over $R$, namely

$$
\mathrm{P}_{k}^{R}(t)=\prod_{i=0}^{\infty} \frac{\left(1+t^{2 i+1}\right)^{\varepsilon_{2 i+1}(R)}}{\left(1-t^{2 i+2}\right)^{\varepsilon_{2 i+2}(R)}}
$$

where $\varepsilon_{j}(R)=\operatorname{rank}_{K} \pi^{j}(R)$ is the $j$ th deviation of $R$. As $\varepsilon_{1}(R)=|\boldsymbol{x}|$, we have

Corollary 2. There is an equality of of formal power series

$$
\frac{\mathrm{P}_{k}^{R}(t)}{(1+t)^{|\boldsymbol{x}|}}=\frac{\mathrm{P}_{k^{\prime}}^{R^{\prime}}(t)}{(1+t)^{\left|\boldsymbol{x}^{\prime}\right|}} .
$$

There always is a coefficientwise inequality of formal power series

$$
\mathrm{P}_{k}^{R}(t) \preccurlyeq \frac{(1+t)^{|x|}}{1+t-t \mathrm{P}_{R}^{S}(t)} .
$$

The ring $R$ is called Golod if equality holds. It is easy to see that $\mathrm{P}_{R}^{S}(t)$ is determined by $L_{I}$, cf. Remark 9 , so from Corollary 2 we recover $[\mathbf{1 2},(3.5 .2)]$, one of the main results of that paper. It can also be obtained directly from Theorem 1 , since by [4] the ring $R$ is Golod if and only if the Lie algebra $\pi^{\geqslant 2}(R)$ is free.

Corollary 3. The ring $R$ is Golod if and only if the ring $R^{\prime}$ is Golod. 
The field $K$ is fixed in the results above. They imply that finitely many series

$$
b_{M, K}(t)=\frac{(1+t)^{|\boldsymbol{x}|}}{\mathrm{P}_{k}^{K[\boldsymbol{x}] /(M)}(t)} \in \mathbb{Z}[[t]]
$$

are obtained by letting $\boldsymbol{x}$ vary over all finite sets of indeterminates and $M$ over all subsets of $n$ monomials. Next we consider what happens when $K$ varies.

Deviations being invariant under field extensions of $K$, Formula (1) shows that the finiteness property still holds when $K$ is allowed to range over all fields of equal characteristic. However, even when the sets $\boldsymbol{x}$ and $M$ are fixed, the polynomial $b_{M, K}(t)$ may change with the characteristic of $K$, see Remark 13 . To analyze the dependence on the characteristic of $K$ we use an important result of Backelin [7], who proves that $b_{M, K}(t)$ is actually a polynomial in $\mathbb{Z}[t]$. In Section 3 we prove

Theorem 4. Let $n$ be a natural number. If $K$ ranges over all fields, $\boldsymbol{x}$ ranges over all finite sets of indeterminates, and $M$ ranges over all subsets of $n$ monomials in $K[\boldsymbol{x}]$, then there exist only finitely many polynomials $b_{M, K}(t)$.

In Section 4 we show that the hypotheses of results above cannot be weakened significantly, nor can their conclusions be strengthened substantially.

\section{Graded Lie algebras}

The arguments depend on DG (= differential graded) homological algebra. Here DG algebras are non-negatively graded and graded-commutative. Taylor DG algebra resolutions of monomial ideals play a central role. We recall their definition, using notation introduced above and referring for details to $[\mathbf{1 0}, \S 5]$.

Fixing a linear order $\succ$ on $M_{I}$, for every two subsets $P, Q$ of $M_{I}$ we set

$$
\operatorname{inv}(P, Q)=|\{(p, q) \in P \times Q \mid p \succ q\}| .
$$

For each $n \in \mathbb{Z}$, let $T_{n}$ be the free $S$-module with basis $\left\{e_{P}\left|P \subseteq M_{I},\right| P \mid=n\right\}$ and let $\partial_{n}: T_{n} \rightarrow T_{n-1}$ be the $S$-linear homomorphism given on $e_{P}$ by

$$
\partial\left(e_{P}\right)=\sum_{p \in P}(-1)^{\operatorname{inv}(\{p\}, P \backslash\{p\})} \frac{m_{P}}{m_{P \backslash\{p\}}} e_{P \backslash\{p\}} .
$$

The pair $(T, \partial)$ is a free resolution of $S / I$ over $S$. The product of $T$ is defined by

$$
e_{P} \cdot e_{Q}= \begin{cases}(-1)^{\operatorname{inv}(P, Q)} \operatorname{gcd}\left(m_{P}, m_{Q}\right) e_{P \cup Q} & \text { if } P \cap Q=\varnothing ; \\ 0 & \text { otherwise }\end{cases}
$$

on pairs of basis elements, and extended to all of $T$ by bilinearity. Different choices of the linear order $\succ$ among the generators of $I$ lead to isomorphic DG algebras.

A $D G \Gamma$-algebra is a DG algebra with a system of divided powers operations $y \mapsto y^{(r)}$, defined for every $y$ of even positive degree and all $r \in \mathbb{N}$, and compatible with the differential. A complete list of the identities they satisfy can be found in $[13,(1.7 .1),(1.8 .1)]$. Morphisms of DG $\Gamma$-algebras commute with all their structures. 
Remark 5. If $U$ and $V$ are DG $\Gamma$-algebras over $S$, then $U \otimes_{S} V$ has a structure of DG $\Gamma$-algebra, functorial in $U$ and $V$ and defined uniquely by the condition that the canonical maps $U \rightarrow U \otimes_{S} V \leftarrow V$ are morphisms, cf. e.g. [13, (1.8.3)]. Every DG algebra over $\mathbb{Q}$ is a $\Gamma$-algebra, with $y^{(r)}=y^{r} /(r !)$, cf. $[\mathbf{1 3},(1.7 .2)]$.

Lemma 6. Every Taylor $D G$ algebra resolution $T$ has a unique structure of $D G$ $\Gamma$-algebra, given for each $n \in 2 \mathbb{N} \backslash\{0\}$ and all $r \in \mathbb{N}$ by the formula

$$
\left(\sum_{P \subseteq M_{I} ;|P|=n} a_{P} e_{P}\right)^{(r)}=\sum_{P_{h} \subseteq M_{I} ;\left|P_{h}\right|=n} a_{P_{1}} \cdots a_{P_{r}} e_{P_{1}} \cdots e_{P_{r}} .
$$

Proof. Let $\gamma_{r}\left(\sum_{P} a_{P} e_{P}\right)$ denote the right hand side of the desired formula. For each element $e_{P}$ the multiplication table (5) gives $e_{P}^{q}=0$ for all $q \geqslant 2$. All elements $e_{P}$ under consideration have even degree, so they commute with each other, hence

$$
\left(\sum_{P} a_{P} e_{P}\right)^{r}=\sum_{q_{1}+\cdots+q_{r}=r} \frac{r !}{\left(q_{1} !\right) \cdots\left(q_{r} !\right)} a_{P_{1}}^{q_{1}} \cdots a_{P_{r}}^{q_{r}} e_{P_{1}}^{q_{1}} \cdots e_{P_{r}}^{q_{r}}=r ! \gamma_{r}\left(\sum_{P} a_{P} e_{P}\right)
$$

To prove existence we vary $K$, so (temporarily) we set $S_{K}=K\left[x_{1}, \ldots, x_{e}\right]$ and let $T_{K}$ denote a Taylor DG algebra resolution of $S_{K} / M_{I} S_{K}$ over $S_{K}$. In $T_{\mathbb{Q}}$ the equality above yields $\gamma_{r}(y)=y^{r} /(r !)$ for all $y \in T_{n}$; by Remark 5 this transforms $T_{\mathbb{Q}}$ into a DG $\Gamma$-algebra. The canonical map $T_{\mathbb{Z}} \rightarrow T_{\mathbb{Z}} \otimes_{\mathbb{Z}} \mathbb{Q}=T_{\mathbb{Q}}$ is injective and commutes with the maps $y \mapsto \gamma_{r}(y)$; they form a system of divided powers on $T_{\mathbb{Q}}$, hence on $T_{\mathbb{Z}}$ as well. By Remark 5 the system of divided powers on $T_{\mathbb{Z}}$ induces one on $T_{K}$ through the isomorphism $T_{K} \cong T_{\mathbb{Z}} \otimes_{\mathbb{Z}} K$ of DG algebras over $K$.

To prove uniqueness, for each $P \subseteq M_{I}$ we set $d_{P}=\operatorname{gcd}\{p \mid p \in P\}$. From the formula for divided powers of a product of elements of even degrees, Equation (5), and the formula for divided powers of a product of elements of odd degrees, we get

$$
d_{P}^{r} e_{P}^{(r)}=\left(d_{P} e_{P}\right)^{(r)}=\left( \pm \prod_{p \in P} e_{\ell}\right)^{(r)}=0
$$

for all $r \geqslant 2$. As $d_{P}$ is not a zero-divisor on $T$, it follows that $e_{P}^{(r)}=0$, hence

$$
\left(\sum_{P} a_{P} e_{P}\right)^{(r)}=\sum_{q_{1}+\cdots+q_{r}=r} a_{P_{1}}^{q_{1}} \cdots a_{P_{r}}^{q_{r}} e_{P_{1}}^{\left(q_{1}\right)} \cdots e_{P_{r}}^{\left(q_{r}\right)}=\gamma_{r}\left(\sum_{P} a_{P} e_{P}\right)
$$

due to the formulas for divided powers of sums and of products.

Homotopy Lie algebras for DG $\Gamma$-algebras are introduced in $[\mathbf{3}]$, cf. also [6].

Remark 7. Let $D$ be a DG $\Gamma$-algebra such that the ring $D_{0}$ is noetherian and the $D_{0}$-module $\mathrm{H}_{n}(D)$ is finite for every $n \in \mathbb{Z}$. If $k$ is a field and $D \rightarrow k$ is a surjective morphism of DG algebras, then the Eilenberg-Moore extension functors define a graded algebra $\operatorname{Ext}_{D}(k, k)$. It is the universal enveloping algebra of a graded Lie algebra $\pi^{\bullet}(D)$, and $D \mapsto \pi^{\bullet}(D)$ is a contravariant functor from the category of DG $\Gamma$-algebras augmented to $k$, to the category of graded Lie algebras over $k$. If $\delta: D \rightarrow D^{\prime}$ is a morphism with $\mathrm{H}_{\bullet}(\delta)$ bijective, then $\pi^{\bullet}(\delta)$ is an isomorphism. 
The morphism $D \rightarrow k$ can be factored as a composition $D \rightarrow U \rightarrow k$ of morphisms of DG $\Gamma$-algebra, such that the underlying graded module of $U$ is free over the underlying graded algebra of $D$, and $\mathrm{H}_{0}(U)=k$. Furthermore, the divided powers of the DG $\Gamma$-algebra $U \otimes_{D} k$ of Remark 5 are inherited by the homology algebra $\mathrm{H}_{\bullet}\left(U \otimes_{D} k\right)$. Let $\mathrm{Q}^{\gamma} \mathrm{H}_{\bullet}\left(U \otimes_{D} k\right)$ denote the residue of $\mathrm{H}_{\bullet}\left(U \otimes_{D} k\right)$ modulo its subspace spanned by the elements of degree 0 , all products of elements of positive degree, and all divided powers of elements of even positive degree. The vector space $\pi^{\bullet}(D)$ is the graded $k$-dual of $\mathrm{Q}^{\gamma} \mathrm{H} \cdot\left(U \otimes_{D} k\right)$.

The next result is part of $[\mathbf{2},(5.1)]$. The proof there is difficult, because the general properties of homotopy Lie algebras discussed above were not available at the time. We provide a short argument, valid for general graded rings $R$.

Lemma 8. Let $E$ be the Koszul complex on $\boldsymbol{x}$, set $D=R \otimes_{S} E$, and give $D$ the $D G \Gamma$-algebra structure described in Remark 5 . If $\iota: R \rightarrow D$ is the inclusion, then $\pi^{\bullet}(\iota)$ yields an isomorphism $\pi^{\bullet}(D) \cong \pi^{\geqslant 2}(R)$ of graded Lie algebras.

Proof. The image of $\boldsymbol{x}$ minimally generates $R$ over $K$, so by a well known theorem of Gulliksen and Schoeller, cf. $[\mathbf{1 3},(1.6 .4)]$ or $[\mathbf{5},(6.3 .5)]$, a factorization $D \rightarrow U \rightarrow k$ as in Remark 7 can be chosen so that $U_{\leqslant 1}=D_{\leqslant 1}$ and $\partial(U) \subseteq(\boldsymbol{x}) U$. The DG algebras $U \otimes_{R} k$ and $U \otimes_{D} k$ then have trivial differentials, so the morphism

$$
\mathrm{H}_{\bullet}\left(U \otimes_{\iota} k\right): \mathrm{H}_{\bullet}\left(U \otimes_{R} k\right) \longrightarrow \mathrm{H}_{\bullet}\left(U \otimes_{D} k\right)
$$

of DG $\Gamma$-algebras is equal to the surjection $U \otimes_{\iota} k: U \otimes_{R} k \rightarrow U \otimes_{D} k$ whose kernel is generated by $U_{1} \otimes_{R} k$. It induces an exact sequence of graded vector spaces

$$
0 \longrightarrow U_{1} \otimes_{R} k \longrightarrow \mathrm{Q}^{\gamma}\left(U \otimes_{R} k\right) \stackrel{\mathrm{Q}^{\gamma}\left(U \otimes_{\iota} k\right)}{\longrightarrow} \mathrm{Q}^{\gamma}\left(U \otimes_{D} k\right) \longrightarrow 0
$$

Since $\pi^{\bullet}(\iota)$ is the $k$-linear dual of $\mathrm{Q}^{\gamma}\left(U \otimes_{\iota} k\right)$, we are done.

The following remark is taken from the proofs of $[\mathbf{1 2},(3.1),(3.5 .1)]$.

Remark 9. Let $I^{\prime}$ be a monomial ideal in the polynomial ring $S^{\prime}=K\left[\boldsymbol{x}^{\prime}\right]$. Assume there exists an isomorphism $\lambda: L_{I} \rightarrow L_{I^{\prime}}$ of LCM lattices. Their atoms are the monomials in $M_{I}$ and $M_{I^{\prime}}$, so $\lambda$ maps $M_{I}$ bijectively onto $M_{I^{\prime}}$; we extend $\lambda$ to a bijection $\widehat{\lambda}$ of the Boolean lattice of $M_{I}$ onto that of $M_{I^{\prime}}$. Let $T^{\prime}$ be the Taylor DG algebra resolution of $S^{\prime} / I^{\prime}$ constructed using the linear order on $M_{I^{\prime}}$ induced from $M_{I}$ via $\lambda$. Formula (4) shows that the map $e_{P} \otimes 1 \mapsto e_{\widehat{\lambda}(P)} \otimes 1$ defines an isomorphism of complexes of vector spaces over $K$; in particular, $\mathrm{P}_{R}^{S}(t)=\mathrm{P}_{R^{\prime}}^{S^{\prime}}(t)$.

If, in addition, $\lambda$ is also an isomorphism of GCD graphs, then Formula (5) shows that $\bar{\lambda}$ is an isomorphism of DG algebras.

Proof of Theorem 1. Let $E$ and $T$ denote Taylor DG algebra resolutions of $k=$ $S /(\boldsymbol{x})$ and $R=S / I$, with augmentations $\varepsilon: E \rightarrow k$ and $\tau: T \rightarrow R$, respectively. By Lemma 6 and Remark 5, the following maps are morphisms of DG $\Gamma$-algebas:

$$
R \otimes_{S} E \stackrel{\tau \otimes_{S} E}{\longleftarrow} T \otimes_{S} E \stackrel{T \otimes_{S} \varepsilon}{\longrightarrow} T \otimes_{S} k .
$$


It is well known that $\mathrm{H}_{\bullet}\left(\tau \otimes_{S} T\right)$ and $\mathrm{H}_{\bullet}\left(T \otimes_{S} \varepsilon\right)$ are isomorphisms. This explains the second and third isomorphisms of graded Lie algebras in the sequence

$$
\pi^{\geqslant 2}(R) \cong \pi^{\bullet}\left(R \otimes_{S} E\right) \cong \pi^{\bullet}\left(T \otimes_{S} E\right) \cong \pi^{\bullet}\left(T \otimes_{S} k\right)
$$

where the first isomorphism is provided by Lemma 8 .

If $T^{\prime}$ is a Taylor DG algebra resolution of $R^{\prime}=S^{\prime} / I^{\prime}$, and $E^{\prime}$ the Koszul complex on $\boldsymbol{x}^{\prime}$, then by symmetry we obtain isomorphisms of graded Lie algebras

$$
\pi^{\bullet}\left(T^{\prime} \otimes_{S^{\prime}} k^{\prime}\right) \cong \pi^{\bullet}\left(E^{\prime} \otimes_{S^{\prime}} T^{\prime}\right) \cong \pi^{\bullet}\left(R^{\prime} \otimes_{S^{\prime}} E^{\prime}\right) \cong \pi^{\geqslant 2}\left(R^{\prime}\right) .
$$

By Formula (6), the isomorphism of DG algebras $\bar{\lambda}: T \otimes_{S} k \rightarrow T^{\prime} \otimes_{S^{\prime}} k^{\prime}$ from Remark 9 commutes with divided powers, so it induces an isomorphism

$$
\pi^{\bullet}\left(T \otimes_{S} k\right) \cong \pi^{\bullet}\left(T^{\prime} \otimes_{S^{\prime}} k^{\prime}\right) .
$$

Assembling the sequences of isomorphisms of graded Lie algebras displayed above, we get the desired isomorphism of graded Lie algebras:

$$
\pi^{\geqslant 2}(R) \cong \pi^{\geqslant 2}\left(R^{\prime}\right) .
$$

This completes the proof of the first assertion of Theorem 1. The second assertion follows immediately from the first one and the elementary remark below.

Remark 10. Let $I$ be a monomial ideal in $K[\boldsymbol{x}]$, minimally generated by $n$ monomials. By construction, the number of vertices in the GCD graph $G_{I}$ is equal to the cardinality of the LCM lattice $L_{I}$, and $\left|L_{I}\right| \leqslant 2^{n}$. Thus, letting $K, \boldsymbol{x}$, and $I$ vary, while keeping $n$ fixed, one obtains only a finite number of pairs $\left(L_{I}, G_{I}\right)$.

\section{Poincaré series}

In this section it is convenient to refocus from ideals generated by monomial ideals to the minimal sets of generators of such ideals.

We identify the polynomial ring $S=K[\boldsymbol{x}]$ and the semigroup $K$-algebra of the free commutative monoid $[\boldsymbol{x}]$ generated by $\boldsymbol{x}$; in particular, we refer to the elements of $[\boldsymbol{x}]$ as monomials in $\boldsymbol{x}$. An antichain in $[\boldsymbol{x}]$ is a finite set $M$ of monomials in the variables $\boldsymbol{x}$ with the property that no $p \in M$ divides any $q \in M \backslash\{p\}$. The map $I \mapsto M_{I}$ is a bijection between the monomial ideals in $S$ and the antichains in $[\boldsymbol{x}]$.

Let $M$ be an antichain in $[\boldsymbol{x}]$. Fix an order $x_{1}, \ldots, x_{e}$ on the indeterminates $\boldsymbol{x}$, and for each $\boldsymbol{i}=\left(i_{1}, \ldots, i_{e}\right) \in \mathbb{N}^{e}$ set $\boldsymbol{x}^{i}=x_{1}^{i_{1}} \cdots x_{e}^{i_{e}}$. Give $S=K[\boldsymbol{x}]$ the standard $\mathbb{N}^{e}$-grading defined by $\operatorname{Deg}\left(\boldsymbol{x}^{i}\right)=\boldsymbol{i}$. This multigrading is inherited by the residue rings $R=S /(M)$ and $k=S /(\boldsymbol{x})$. For every $\mathbb{N}^{e}$-graded $R$-module $N$ and each $j \in \mathbb{N}$ the finite $K$-vector space $\operatorname{Ext}_{R}^{j}(N, k)$ is multigraded. The series

$$
\mathrm{P}_{N}^{R}(\boldsymbol{s}, t)=\sum_{i \in \mathbb{N}^{e} ; j \in \mathbb{N}} \operatorname{rank}_{K} \operatorname{Ext}_{R}^{j}(N, k)^{i} \boldsymbol{s}^{i} t^{j} \in \mathbb{Z}\left[\boldsymbol{s}^{ \pm 1}\right][[t]]
$$

is the multigraded Poincaré series of $N$. Setting $\mathbf{1}=(1, \ldots, 1) \in \mathbb{N}^{e}$, one recovers the Poincaré series in one variable by means of the formula

$$
\mathrm{P}_{N}^{R}(t)=\mathrm{P}_{N}^{R}(\mathbf{1}, t) .
$$


Backelin [7] proves that the multigraded Poincaré series satisfies the condition

$$
b_{M, K}(\boldsymbol{s}, t) \cdot \mathrm{P}_{k}^{R}(\boldsymbol{s}, t)=\left(1+s_{1} t\right) \cdots\left(1+s_{e} t\right)
$$

for some polynomial $b_{M, K}(s, t) \in \mathbb{Z}[s, t]$ subject to the following restrictions:

$$
\begin{gathered}
\operatorname{deg}_{s_{h}}\left(b_{M, K}(s, t)\right) \leqslant \max \left\{\operatorname{deg}_{x_{h}}(p) \mid p \in M\right\} \text { for } h=1, \ldots, e ; \\
\operatorname{deg}_{t}\left(b_{M, K}(\boldsymbol{s}, t)\right) \leqslant \operatorname{deg}(\operatorname{lcm}\{p \in M\}) .
\end{gathered}
$$

We now head for a proof of Theorem 4, dealing with Poincaré series $\mathrm{P}_{k}^{K[\boldsymbol{x}] /(M)}(t)$ for antichains of monomials $M$ in $[\boldsymbol{x}]$. The following elementary fact will be needed.

Lemma 11. If $M$ is a fixed antichain $M$ and $K$ ranges over all fields, then there exist only finitely many Poincaré polynomials $\mathrm{P}_{K[\boldsymbol{x}] /(M)}^{K[\boldsymbol{x}]}(\boldsymbol{s}, t) \in \mathbb{Z}[\boldsymbol{s}, t]$.

Proof. Let $T$ denote the Taylor resolution of $\mathbb{Z}[\boldsymbol{x}] /(M)$ over $\mathbb{Z}[\boldsymbol{x}]$. Since $T \otimes_{\mathbb{Z}} K$ is the Taylor resolution of $K[\boldsymbol{x}] /(M)$ over $K[\boldsymbol{x}]$, the isomorphism

$$
\operatorname{Hom}_{\mathbb{Z}[\boldsymbol{x}]}(T, \mathbb{Z}) \otimes_{\mathbb{Z}} K \cong \operatorname{Hom}_{K[\boldsymbol{x}]}\left(\left(T \otimes_{\mathbb{Z}} K\right), k\right)
$$

of complexes of multigraded $K$-spaces yields for all $j \in \mathbb{Z}$ and $\boldsymbol{i} \in \mathbb{N}^{e}$ isomorphisms

$$
\operatorname{Ext}_{K[\boldsymbol{x}]}^{j}(K[\boldsymbol{x}] /(M), k)^{i} \cong \mathrm{H}_{-j}\left(\operatorname{Hom}_{\mathbb{Z}[\boldsymbol{x}]}(T, \mathbb{Z}) \otimes_{\mathbb{Z}} k\right)^{i} .
$$

Let $\mathcal{P}$ be the set of prime numbers that annihilate some non-zero homology class of $\operatorname{Hom}_{\mathbb{Z}[\boldsymbol{x}]}(T, \mathbb{Z})$. For $\operatorname{char}(K) \notin \mathcal{P}$ the Künneth formula yields isomorphisms

$$
\mathrm{H}_{-j}\left(\operatorname{Hom}_{\mathbb{Z}[\boldsymbol{x}]}(T, \mathbb{Z}) \otimes_{\mathbb{Z}} k\right)^{i} \cong\left(\mathrm{H}_{-j}\left(\operatorname{Hom}_{\mathbb{Z}[\boldsymbol{x}]}(T, \mathbb{Z})\right) \otimes_{\mathbb{Z}} k\right)^{\boldsymbol{i}}
$$

for all $j$ and $\boldsymbol{i}$. Thus, the polynomials $\mathrm{P}_{K[\boldsymbol{x}] /(M)}^{K[\boldsymbol{x}]}(\boldsymbol{s}, t)$ are equal for all $K$ with $\operatorname{char}(K) \notin \mathcal{P}$. To finish the proof, it remains to remark that $\mathcal{P}$ is finite, because $\operatorname{Hom}_{\mathbb{Z}[\boldsymbol{x}]}(T, \mathbb{Z})$ is a finite complex of finitely generated free abelian groups.

Proof of Theorem 4. Let $n$ be a natural number. By Remark 10 we may choose a finite family $\mathcal{F}_{n}$ of antichains of $n$ monomials with the following property: All pairs $\left(L_{I^{\prime}}, G_{I^{\prime}}\right)$ that can be obtained from some ideal $I^{\prime}$ minimally generated by $n$ monomials in some polynomial ring over some field $K$ have the form $\left(L_{I}, G_{I}\right)$ for some $I=(M) K[\boldsymbol{x}]$ with $M \in \mathcal{F}_{n}$. Thus, we obtain

$$
\sup _{K} \sup _{\boldsymbol{x}} \sup _{|M|=n} \operatorname{deg}\left(b_{M, K}(t)\right)=\sup _{K} \sup _{M \in \mathcal{F}_{n}} \operatorname{deg}\left(b_{M, K}(t)\right) \leqslant \sup _{M \in \mathcal{F}_{n}} \operatorname{deg}\left(m_{M}\right)<\infty
$$

where the equality comes from Formula (3) and Corollary 2, the first inequality from Formula (9), and the second inequality from the finiteness of $\mathcal{F}_{n}$. Choose a natural number $d$ such that $\operatorname{deg}\left(b_{M, K}(t)\right) \leqslant d$ for all $M$ and all $K$.

Fix, for the moment, an antichain $M$ and a field $K$. Since $b_{M, K}(0)=1$, there is a unique decomposition $b_{M, K}(t)=\prod_{r=0}^{d}\left(1-\beta_{r} t\right)$ with $\beta_{r} \in \mathbb{C}$. Let $\beta_{M, K}$ denote the maximal absolute value of these complex numbers, and let $\rho_{M, K}$ denote the radius of convergence of the rational function $(1+t)^{|\boldsymbol{x}|} /\left(1+t-t \mathrm{P}_{K[\boldsymbol{x}] /(M)}^{K[\boldsymbol{x}]}(t)\right)$. As Formula 
(3) shows that $1 / \beta_{M, K}$ is equal to the radius of convergence of the Poincare series $\mathrm{P}_{k}^{K[\boldsymbol{x}] /(M)}(t)$, we obtain $1 / \beta_{M, K} \geqslant \rho_{M, K}$ by Formula $(2)$.

On the other hand, Formula (3) and Corollary 2 yield

$$
\inf _{K} \inf _{\boldsymbol{x}} \inf _{|M|=n}\left(\rho_{M, K}\right)=\inf _{K} \inf _{M \in \mathcal{F}_{n}}\left(\rho_{M, K}\right) .
$$

Since $\mathcal{F}_{n}$ is finite, Lemma 11 shows that the second infimum can be computed by using only finitely many fields $K$, hence it is equal to a real number $\rho>0$.

Summing up, we obtain inequalities $\beta_{M, K} \leqslant 1 / \rho<\infty$ for every choice of $M$ and $K$. They imply that in every polynomial $b_{M, K}(t)$ the absolute value of the coefficient of $t^{r}$ does not exceed $\left(\begin{array}{c}d \\ r\end{array}\right) / \rho^{r}$. Since each $b_{M, K}(t)$ has integer coefficients, there is only a finite number of distinct polynomials of this form.

\section{Discussion}

We start with a problem suggested by Theorem 4 .

Remark 12. Theorem 4 may be restated as asserting the finiteness of the number

$$
d(n)=\sup _{K} \sup _{\boldsymbol{x}} \sup _{|M|=n} \operatorname{deg}\left(b_{M, K}(t)\right) .
$$

In view of Formula (3), the ranks of the modules $F_{j}$ for $j=0, \ldots, d(n)$ in a minimal free resolution $F$ determine the entire Poincaré series of $k$ over $K[\boldsymbol{x}] /(M)$. Thus, knowledge of an effective bound on $d(n)$ would be extremely useful for computational purposes. Using the sets $M=\left\{x^{2} \mid x \in \boldsymbol{x}\right\}$ one gets $d(n) \geqslant 2 n$. Equality is easy to check for $n \leqslant 3$. For all $n$, it would be consistent with observations of Charalambous and Reeves [9, p. 2390] based on computer experiments.

It is known that the characteristic of $K$ may affect the Poincaré series of $k$. To document this fact we present a construction proposed by the referee.

Remark 13. The polynomial $b_{M, K}(t)$ may vary with the characteristic of $K$. Indeed, let $M^{\prime}$ be an antichain of monomials, fix an indeterminate $x \in \boldsymbol{x}$, and consider the antichain $M=x M^{\prime}$. For every field $K$, the ring $K[\boldsymbol{x}] /(M)$ is Golod by a theorem of Shamash [17], so the inequality in Formula (2) becomes an equality. In view of Formula (7), it suffices to show that the Poincaré series $\mathrm{P}_{K[\boldsymbol{x}] /(M)}^{K[\boldsymbol{x}]}(t)$ depends on $K$. It is equal to $\mathrm{P}_{K[\boldsymbol{x}] /\left(M^{\prime}\right)}^{K[\boldsymbol{x}]}(t)$, so examples may be obtained by choosing $M^{\prime}$ to be the squarefree monomial ideal associated to a simplicial complex $\Delta$, whose reduced homology groups $\widetilde{\mathrm{H}}_{i}(\Delta, K)$ vary with the characteristic of $K$, cf. [14].

The preceding examples notwithstanding, some terms of $b_{M, K}(s, t)$ are independent of $K$. This is shown by the remark that follows, which also shows that the degree bounds in Formula (8) are in fact equalities.

Remark 14. Every antichain $M$ in $[\boldsymbol{x}]$ is determined by any one of the polynomials $b_{M, K}(s, t)$ for some $K$ through the congruence 


$$
b_{M, K}(\boldsymbol{s}, t) \equiv 1-\left(\sum_{p \in M} s^{\operatorname{Deg}(p)}\right) t^{2} \bmod \left(t^{3} \mathbb{Z}[\boldsymbol{s}, t]\right) .
$$

Indeed, standard equalities, cf. e.g. [5, (7.1.5) and (7.1.1)], yield a congruence

$$
\mathrm{P}_{k}^{R}(s, t) \equiv 1+\left(\sum_{h=1}^{e} s_{i}\right) t+\left(\sum_{1 \leqslant g<h \leqslant e} s_{g} s_{h}+\sum_{p \in M} s^{\operatorname{Deg}(p)}\right) t^{2} \quad \bmod \left(t^{3} \mathbb{Z}[\boldsymbol{s}][[t]]\right) .
$$

Writing $b_{M, K}(\boldsymbol{s}, t)$ in the form $\sum_{n} p_{n}(\boldsymbol{s}) t^{n}$ with $p_{n}(\boldsymbol{s}) \in \mathbb{Z}[\boldsymbol{s}]$, we get the desired congruence by comparing the coefficients of $1, t$, and $t^{2}$ in Equality (7).

The remark above produces infinitely many polynomials $b_{M, K}(s \mathbf{1}, t)$ in two variables. Combining this information with Formulas (7) and (1), we obtain

Remark 15. When $I$ varies over all ideals of $S$ generated by a fixed number of monomials there exist infinitely many power series in two variables of the form $\mathrm{P}_{k}^{S / I}(s \mathbf{1}, t)$; as a consequence, the isomorphism of Lie algebras in Theorem 1 does not preserve gradings - let alone multigradings - induced by those of $S$.

Finally, we emphasize that the finiteness conclusions of Theorems 1 and 4 heavily depend on the hypothesis that the ideals involved are generated by monomials.

Remark 16. When $J$ varies over all ideals of $S$ generated by a fixed number of forms of prescribed degrees, the number of power series $\mathrm{P}_{k}^{S / J}(t)$ may be infinite, and hence there may exist infinitely many non-isomorphic graded Lie algebras $\pi^{\geqslant 2}(S / J)$.

Indeed, assume $K$ has characteristic 0. Fröberg, Gulliksen, and Löfwall [11] construct a family of ideals $\left\{J_{a} \subset K\left[x_{1}, \ldots, x_{15}\right] \mid a \in K\right\}$ generated by 67 quadratic forms, such that the rings $R_{a}=S / J_{a}$ have the same Hilbert series for all $a$, but there are infinitely many different Poincaré series $\mathrm{P}_{k}^{R_{a}}(t)$. Also, Roos [16] exhibits a family of ideals $\left\{J_{a} \subset K\left[x_{1}, \ldots, x_{6}\right] \mid a \in \mathbb{N}\right\}$ generated by 11 quadratic forms, with equal Hilbert series and different Poincaré series for all $a \geqslant 2$.

\section{Acknowledgements}

I should like to thank the referee for two very close readings of the manuscript, and for suggesting - and insisting on - a detailed treatment of the role of the characteristic of the base field. I also want to thank Jörgen Backelin for useful discussions that influenced the final presentation of this material. 


\section{References}

[1] D. Anick, A counterexample to a conjecture of Serre, Ann. of Math. (2) 115 (1982), 1-33; Comment, ibid. 116 (1982), 661.

[2] L. L. Avramov, The Hopf algebra of a local ring, Izv. Akad. Nauk SSSR Ser. Mat. 38 (1974), 253-277 [Russian; English translation:] Math. USSR-Izv. 38 (1974), 259-284.

[3] L. L. Avramov, Local algebra and rational homotopy, Homotopie Algébrique et Algèbre Locale (Luminy, 1982) Astérisque 113-114, Soc. Math. France, Paris, 1984; pp. 15-43.

[4] L. L. Avramov, Golod homomorphisms, Algebra, algebraic topology, and their interactions (Stockholm, 1983), Lecture Notes Math. 1183, Springer, BerlinNew York, 1986; pp. 58-78.

[5] L. L. Avramov, Infinite free resolutions, in: Six lectures in commutative algebra, Bellaterra, 1996, Progress in Math. 166, Birkhäuser, Boston, 1998; pp. $1-118$.

[6] L. L. Avramov, S. Halperin, Through the looking glass: a dictionary between rational homotopy theory and local algebra, Algebra, algebraic topology and their interactions (Stockholm, 1983), Lecture Notes in Math. 1183, Springer, Berlin-New York, 1986; pp. 1-27.

[7] J. Backelin, Les anneaux locaux à relations monomiales ont des séries de Poincaré-Betti rationnelles, C. R. Acad. Sci. Paris Sér. I Math. 295 (1982), 607-610.

[8] J. Backelin, J.-E. Roos, When is the double Yoneda Ext-algebra of a local Noetherian ring again Noetherian?, Algebra, algebraic topology and their interactions (Stockholm, 1983), Lecture Notes in Math. 1183, Springer, BerlinNew York, 1986; pp. 101-119.

[9] H. Charalambous, A. Reeves, Poincaré series and resolutions of the residue field over monomial rings, Comm. Algebra 23 (1995), 2389-2399.

[10] R. Fröberg, Some complex constructions with applications to Poincaré series, Séminaire d'Algèbre P. Dubreil 31ème année (Paris, 1977-1978), Lecture Notes in Math. 740, Springer, Berlin-New York, 1979; pp. 272-284.

[11] R. Fröberg, T. H. Gulliksen, C. Löfwall, Flat families of local, artinian algebras with an infinite number of Poincaré series, Algebra, algebraic topology and their interactions (Stockholm, 1983), Lecture Notes in Math. 1183, Springer, Berlin-New York, 1986; pp. 170-191.

[12] V. Gasharov, I. Peeva, V. Welker, The LCM-lattice in monomial resolutions, Math. Res. Letters 6 (1999), 521-532.

[13] T. H. Gulliksen, G. Levin, Homology of local rings, Queen's Papers Pure Appl. Math. 20, Queen's Univ., Kingston, ON, 1969.

[14] G. A. Reisner, Cohen-Macaulay quotients of polynomial rings, Adv. in Math. 21 (1976), 30-49. 
[15] J.-E. Roos, Relations between Poincaré-Betti series of loop spaces and of local rings, Séminaire d'Algèbre P. Dubreil 31ème année (Paris, 1977-1978), Lecture Notes in Math. 740, Springer, Berlin-New York, 1979; pp. 285-322.

[16] J.-E. Roos, Commutative non Koszul algebras having a linear resolution of arbitrary high order. Applications to torsion in loop space homology, C. R. Acad. Sci. Paris Sér. I 316 (1993), 1123-1128.

[17] J. Shamash, The Poincaré series of a local ring, J. Algebra 12 (1969), 453470 .

This article may be accessed via WWW at http://www.rmi.acnet.ge/hha/ or by anonymous ftp at

ftp://ftp.rmi.acnet.ge/pub/hha/volumes/2002/n2a1/v4n2a1.(dvi,ps,pdf)

Luchezar L. Avramov avramov@math.unl.edu

Department of Mathematics,

Purdue University,

West Lafayette, IN 47907,

USA 